\title{
Simulación clínica, una herramienta en tiempos de crisis
}

\author{
Clinical simulation, a tool in times of crisis
}

\section{Eliana Escudero Z*}

\section{INTRODUCCIÓN}

$\mathrm{D}^{\mathrm{s}}$ esde inicios del 2020, el mundo enfrenta la pandemia por COVID-19 que ha mostrado dolor, temor, incertidumbre, pérdidas, daños económicos y grandes dificultades para dar continuidad a la vida que estábamos acostumbrados a realizar. ${ }^{1}$ Las cifras alarmantes de contagiados y fallecidos levantan una serie de cuestionamientos respecto a cómo resolver temas sanitarios y controlar la grave situación, pero también cómo preparar a nuestros profesionales de salud para abordar esta terrible emergencia sanitaria que se ha convertido en uno de los mayores problemas de salud pública de los últimos tiempos. ${ }^{2,3}$ Adquirir las competencias con nuevas modalidades y alcanzar la calidad que se requiere es un desafío desde muchos ámbitos, implica creatividad, flexibilidad, seguridad y modificar los modelos tradicionales de enseñanza, incorporando la multidisciplinariedad, las tecnologías, el trabajo colaborativo y especialmente la innovación. ${ }^{2}$ La seguridad del paciente está en riesgo elevado, con un personal de salud afectado, cansado, con un sistema que exige adaptarse a nuevos roles, procesos, tecnologías y con equipos de trabajo de poca experiencia. La crisis del sistema se convierte en una oportunidad y el desafío de tomar el liderazgo de los profesionales de salud es prioritario y urgente para vencer las grandes dificultades que toca resolver. ${ }^{3}$

Pareciera que tenemos dos grandes desafíos, el primero corresponde al ámbito de todo lo que tiene relación con las acciones específicas para apoyar a los profesionales de primera línea y reflexionar sobre la influencia del factor humano en los resultados de sus desempeños y proponer cambios que busquen desarrollar una cultura de seguridad. ${ }^{4}$ El segundo es cómo llevar a cabo la formación de pregrado y postítulo o especialidades, cómo planificar los semestres, las prácticas clínicas y las evaluaciones para certificar a los estudiantes y concluir los procesos formativos con calidad y con una nueva normalidad de la enseñanza. ${ }^{5}$

Las inquietudes, la incertidumbre y las discusiones son variadas y no es fácil dar una sola respuesta; sin embargo, la comunidad de simulación en el mundo se ha unido para dar soluciones y propuestas, sobreponiéndose a todas las circunstancias y haciendo un llamado a repensar el rol de los simulacionistas y los centros de simulación. ${ }^{6}$

Por ello, pareciera valioso compartir un pequeño resumen de lo que está ocurriendo a un año de pandemia y cuáles son los aprendizajes y buenas experiencias que han permitido avanzar gracias al aporte de la educación basada en simulación (EBS).

\section{EL ROL DE LAS ORGANIZACIONES Y SOCIEDADES CIENTÍFICAS}

Gaba en el 2007 señaló que el logro de la mejora en la seguridad del paciente depende del trabajo que realice cada miembro de las comunidades de simulación. ${ }^{7}$ Es así como hemos visto el gran compromiso de las sociedades científicas desde los inicios de la pandemia, haciendo una continua y permanente contribución a la comunidad clínica y de educación con una gran preocupación por el profesional de primera línea y por las instituciones de educación de carreras de salud.

La Society for Simulation in Healthcare (SSH) cuenta con un sitio denominado COVID-19 Update, ${ }^{8}$ en el cual se dispone de diversos recursos como eventos, videos, soluciones tecnológicas y publicaciones sobre simulación. El trabajo de los miembros del board, sus comités y grupos de in-
* Decana de la
Universidad Diego Portales. Chile.

doi: $10.35366 / 99862$
Citar como: Escudero ZE. Simulación clínica, una herramienta en tiempos de crisis. Rev Latinoam Simul Clin. 2021; 3 (1): 3-6. https://dx.doi.org/10.35366/99862 
terés se mantiene dialogando y discutiendo por las mejores opciones y dar respuesta a las necesidades de los profesionales y educadores de salud. La semana de simulación en septiembre se celebró con más participantes que años anteriores, lo que se puede interpretar como una señal de interés por la temática de la simulación. ${ }^{9}$ Se dispone de un adendum del diccionario de simulación, lo que permite que la taxonomía de la simulación a distancia se comprenda y estandarice en su aplicación. ${ }^{10}$ Transformar el Congreso IMSH 2021, tradicionalmente presencial, por una versión virtual fue tarea difícil, ya que es un hito esperado por miles de personas que asisten cada año para crecer con sus redes de colaboración y encontrar nuevas soluciones y respuestas a sus necesidades. Sin embargo, disponer del material del congreso en una plataforma virtual por casi tres meses ha sido una interesante opción de seleccionar autores, temas o empresas y seguir actualizándose y perfeccionándose (http://imsh2021.org/program).

Es relevante la declaración del 31 de marzo de 2020 de SSH junto a la International Nursing Association for Clinical Simulation and Learning (INACSL), la cual reconoce la homologación de horas de práctica por simulación a distancia. Se proporciona a través de esta declaración un fundamento desde los expertos, para que las instituciones de educación tomen la decisión de incorporar estas nuevas tecnologías a sus actividades de educación basada en simulación (EBS). ${ }^{11}$ Ambas sociedades trabajan de manera colaborativa, esta afirmación fue indirectamente una invitación a la industria, quienes se pusieron a disposición de la academia en el desarrollo de nuevos recursos y otorgaron más accesibilidad, no sólo desde los costos, sino también por la traducción al español de muchos de ellos y el aumento de nuevas opciones.

INACSL, sociedad que también adapta su trabajo a esta nueva realidad, nos proporciona la traducción en español de los estándares de mejores prácticas en simulación. ${ }^{12}$ Disponer de estos estándares y el detalle de cada uno de los documentos, con sus criterios y su evidencia que los respalda, permite a los académicos avanzar hacia la simulación de excelencia.

Por otro lado, la Federación Latinoamericana de Simulación Clínica y Seguridad del Paciente (FLASIC), que une a latinoamericanos e hispanoparlantes del mundo, nos ha mantenido activos a través de su revista y del vínculo con las sociedades que la conforman, ha generado actividades en forma permanente y con el esfuerzo de cada socio podrá seguir contribuyendo para que en esta oportunidad la simulación crezca con la calidad y el reconocimiento que merece. ${ }^{6}$ La integración en el currículum de espacios e iguales oportunidades de aprendizaje para toda la comunidad de salud, con la ética del simulacionista y la cultura de seguridad, depende más que nunca de la comunidad de simulación. 7,13

La Organización Mundial de la Salud (OMS) está presente desde la aparición de los primeros casos de COVID-19 y es relevante mencionar su permanente apoyo a las iniciativas que levanta la alianza mundial para la seguridad del paciente; coincide que el Día Mundial de la Seguridad del Paciente se celebra en la misma semana que celebramos simulación, y el 2020 se reflexionó sobre la crisis de seguridad que hemos vivido, no sólo del paciente, también de todo el equipo de salud, lo que lleva a plantear nuevos desafíos. ${ }^{3}$ En la propuesta de la OMS publicada en enero de 2021 se declara un marco de indicadores para lograr objetivos de seguridad, cabe señalar el acercamiento de la simulación con dos de ellos, en primer lugar el número 5, que se refiere a funcionarios de salud y educadores con una de las siguientes estrategias: "avanzar en el uso de métodos de simulación en todo el sistema de educación y formación profesional, identificando centros para liderar el desarrollo del proceso"; ${ }^{14}$ y el segundo se relaciona con sistemas de alta confianza, es decir, aquello que asociamos con factor humano y que en simulación trabajamos a través de escenarios de Manejo de Recurso en Crisis (CRM por sus siglas en inglés). La OMS está fortaleciendo el trabajo de los educadores de simulación con claridad y actividades muy específicas.

\section{UNIVERSIDADES}

Para las instituciones de educación superior, institutos técnicos-profesionales y universidades, el desafío es trascendental, la adaptación y la rápida respuesta será lo que permita dar solución a los estudiantes y cumplir con los compromisos correspondientes. Muchos académicos y docentes se cuestionan respecto a la calidad de los aprendizajes y experiencias que tal vez no logren las generaciones actuales. Se instala una preocupación que debemos convertir en una oportunidad y dar a la EBS las mejores opciones de eficiencia, eficacia y seguridad, creando en estos espacios nuevos conocimientos y competencias. ${ }^{6,15}$ No se desconoce la necesidad e importancia del encuentro del aprendiz con el ambiente clínico y 
los pacientes; sin embargo, dado que los modelos existentes en salud no cuentan con evidencia al respecto, la problemática se transforma en una oportunidad para investigar y estandarizar. ${ }^{16} \mathrm{La}$ innovación curricular, las adecuaciones de las metodologías y los aprendizajes esperados son tareas de docentes, directivos y vicerrectorías académicas, propiciando el desarrollo de pensamiento crítico, comunicación efectiva y trabajo en equipo, aunque no debemos dejar de mencionar el aporte que ha dado la simulación en los ámbitos clínicos. Para quienes entraron a la primera línea y debieron entrenar técnicas diversas como colocación y retiro de equipamiento de protección personal, el manejo e intubación de paciente y procedimientos aún más críticos, como es el traslado aéreo de pacientes COVID, la simulación ha sido una herramienta fundamental para enfrentar mejor esta gran crisis. ${ }^{17}$ La adaptación de los profesionales a nuevos roles también es algo que emerge en esta pandemia lo que fortalece el concepto de trabajo interdisciplinar colaborativo.

La comunidad de simulación ha visto la creación de nuevos centros, incorporación de la simulación a distancia, el uso de software, escenarios de aula invertida, paciente simulado en virtualidad, telesimulación, etcétera, muchas formas que no estaban presentes antes de la pandemia COVID-19. La capacitación de los equipos docentes en EBS ha aumentado y probablemente seguirá siendo una deuda pendiente el seguir creciendo en el formador de formadores, que aplique estándares de mejores prácticas y así avancemos en la calidad de la simulación.

\section{CONCLUSIONES}

Necesitamos contar con profesionales entrenados y con capacidad de conformar nuevos equipos de trabajo, además de prepararlos para prevenir errores que en situaciones de crisis y en esta pandemia de COVID-19 van en aumento. Se requiere implementar la EBS midiendo su impacto y generando evidencia que dé sustento a estos cambios metodológicos en la formación de pregrado. Generar alianzas y redes de colaboración permite aprender del y con el otro. Es así como surge la creación de un libro, pronto a publicarse, cuya idea nace en un workshop de un congreso y

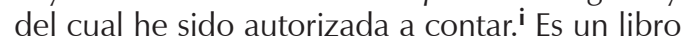

\footnotetext{
i Autorización de editores: Simon Flandin, Christine VidalGomel y Raquel Becerril.
}

que une a Francia, Australia, Italia, Suiza, Reino Unido, Noruega, Bélgica y Chile y que, por sus temáticas y construcción, nos permitirá avanzar y ampliar nuestros conocimientos en simulación; la gran contribución y particularidad del libro es el desarrollo, a lo largo de sus capítulos, de un proceso de integración de todos los ámbitos de la simulación. Es decir, conecta diseño con implementación y aplicación, indagando ámbitos ocultos de cómo se vive la experiencia y los obstáculos del aprendizaje. Une resultados de la experiencia con estudios empíricos, construyendo nueva evidencia. Crecer en nuestros conocimientos y en redes de colaboración nos permitirá salir fortalecidos de esta pandemia.

\section{REFERENCIAS}

1. Paige JT. What's in a name? Simulation and technology enhanced learning uses and opportunities in the era of COVID-19. BMJ Simul Technol Enhanc Learn. 2021; 7 (1): 1-2. http://dx.doi.org/10.1136/ bmjstel-2020-000681

2. Singh $\mathrm{H}$, Sittig DF, Gandhi TK. Fighting a common enemy: a catalyst to close intractable safety gaps. BMJ Qual Saf. 2021; 30 (2): 141-145.

3. Donaldson LJ, Neelam D. World patient safety day: A call for action on health worker safety. J Patient Saf Risk Manag. 2020; 25 (5): 171-173.

4. Weinger MB, Gaba DM. Human factors engineering in patient safety. Anesthesiology. 2014; 120 (4): 801-806.

5. Ingrassia PL, Capogna G, Diaz-Navarro C, Szyld D, Tomola S, Leon-Castelao E. COVID-19 crisis, safe reopening of simulation centres and the new normal: food for thought. Adv Simul (Lond). 2020; 5: 13.

6. Park CS, Clark L, Gephardt G, Robertson JM, Miller J, Downing DK et al. Manifesto for healthcare simulation practice. BMJ Simul Technol Enhanc Learn. 2020; 6: 365-368.

7. Gaba DM. The future vision of simulation in healthcare. Simul Healthc. 2007; 2 (2): 126-35. doi: 10.1097/01. $\mathrm{SIH} .0000258411 .38212 .32$

8. COVID-19 Updates. Available in: https://www.ssih.org/ COVID-19-Updates/-Helpful-Links-and-Information

9. International Simulation Data Registry. Available in: https://www.ssih.org/SSH-Resources/InternationalSimulation-Data-Registry-ISDR

10. Lioce L (Ed.), Lopreiato J, Downing D, Chang TP, Robertson JM, Anderson $\mathrm{M}$ et al. Healthcare simulation. Dictionary. 2nd edition. Rockville, MD: Agency for Healthcare Research and Quality; September 2020. AHRQ Publication No. 20-0019. doi: https://doi.org/10.23970/simulationv2

11. SSH-INACSL. COVID-19: SSH/INACSL position statement on use of virtual simulation during the pandemic. March 31 2021. Available in: https://www. ssih.org/COVID-19-Updates/ID/2237/COVID-19SSHINACSL-Position-Statement-on-Use-of-VirtualSimulation-during-the-Pandemic 
12. Standards of Best Practice. 2021. Available in: https:// www.inacsl.org/inacsl-standards-of-best-practicesimulation/translation-of-standards/

13. Park CS, Murphy TF, the Code of Ethics Working Group. Healthcare simulationist code of ethics. 2018. Available in: http://www.ssih.org/Code-of-Ethics

14. World Health Organization. Global patient safety action plan 2021-2030. Toward eliminating avoidable harm in health care. Geneva: World Health Organization; 2021.

15. McNaughton N, Gormley G. From manifestos to praxis: developing criticality in healthcare simulation. BMJ Simul Technol Enhanc Learn. 2021; 1-3. doi: 10.1136/ bmjstel-2020-000821.
16. Leighton K, Kardong-Edgren S, McNelis AM, Foisy-Doll C, Sullo E. Traditional clinical outcomes in prelicensure nursing education: an empty systematic review. J Nurs Educ. 2021; 60 (3): 136-142.

17. Vera M, Kattan E, Cerda T, Niklitshek J, Montaña $\mathrm{R}$, Varas J et al. Implementation of distancebased simulation training programs for healthcare professionals. Simul Healthc. 2021. doi: 10.1097/ SIH.0000000000000550.

Correspondencia:

Eliana Escudero Z

E-mail: eliana.escudero@udp.cl 